S.M. Berman

Nagoya Math. J.

Vol. 46 (1972), 63-86

\title{
GAUSSIAN SAMPLE FUNGTIONS: UNIFORM DIMENSION AND HÖLDER CONDITIONS NOWHERE
}

\author{
SIMEON M. BERMAN
}

\section{Introduction.}

Let $X(t), t \geq 0$, be a real Gaussian process with mean 0 , stationary increments, and $\sigma^{2}(t)=E|X(t)-X(0)|^{2}$. Here $\sigma^{2}(t)=\int_{-\infty}^{\infty}\left|e^{i \lambda t}-1\right|^{2} \lambda^{-2}\left(1+\lambda^{2}\right)$ $d H(\lambda)$, for some bounded monotone $H$. We summarize the main results. If the derivative $H^{\prime}$ of the absolutely continuous component of $H$ satisfies $H^{\prime}(\lambda) \geq C|\lambda|^{-\alpha-1}$ for all large $|\lambda|$, for some $0<\alpha<2$, then i) The local time $\varphi(x, t)$ of the sample function exists, is jointly continuous in $(x, t)$, and satisfies a uniform Hölder condition in $t$ of any order smaller than $1-\alpha / 2$, almost surely; ii) $X(t), 0 \leq t \leq T$, nowhere satisfies a Hölder condition of order greater than $\alpha / 2$, almost surely. If, furthermore, the sample functions are almost surely continuous, then $\{x: \operatorname{dim}[t: 0 \leq t \leq T, X(t)$ $=x]<1-\alpha / 2\}$ is nowhere dense, almost surely. If, in addition, $\sigma^{2}(t) \leq$ $B|t|^{\beta}, 0 \leq t \leq T$ for some $0<\beta<2$, then $\operatorname{dim}\{t: 0 \leq t \leq T, X(t)=x\} \leq 1-\beta / 2$ for all $x$, almost surely. If $X(t)$ is stationary and ergodic, and $\alpha=\beta$ in the conditions above, then $\operatorname{dim}\{t: t \geq 0, X(t)=x\}=1-\alpha / 2$ for all $x$, almost surely.

The theme of the preceding three papers [3], [4], and [5] is that the smoothness of the local time of a Gaussian process implies the irregularity of the sample functions. Here we continue to demonstrate this implication in a quantitative way, and sharpen some of the earlier results.

The original calculations for the proof of the continuity of the local time of a Gaussian process are in [3]. The conditions were simplified and weakened, and joint continuity was proved in [5]. While not strictly comparable to those in [5], the hypotheses here are more simply stated, and the conclusions are stronger (Theorem 4.1).

Received April 13, 1971.

This paper represents results obtained at the Courant Institute of Mathematical Sciences, New York University, under the sponsorship of the National Science Foundation, Grant NSF -GP-11460. 
Theorem 5.1 states that the sample functions nowhere satisfy a Hölder condition of a certain order. Although the hypothesis is stronger than that in [4], the conclusion is more precise.

The novelty of our results on the dimension of the $x$-values of the sample functions is that they yield an upper bound on the dimension which is uniform in $x$ (Theorem 7.1), and an exact evaluation uniform in $x$ for stationary processes (Theorem 8.1). Our earlier result in [5] and Orey's in [11] give the dimension for a fixed value of $x$. Our present hypothesis is slightly stronger than that of Orey.

It also follows from our method that in the particular case of the Brownian motion process, the exact dimension of the pre-image of every point in the interior of the range is equal to $1 / 2$, almost surely.

A major part of the analytics of Gaussian local times concerns the determinant of the covariance matrix of the finite-dimensional distribution of the process. An elementary inequality for such determinants (Lemma 2.1) is used for the first time; the calculations are simpler than in [3] and [5].

1. Gaussian processes with stationary increments and their local times.

Let $X(t), t \geq 0$, be a Gaussian process with mean 0 and stationary increments. Put $\sigma^{2}(t)=E(X(t)-X(0))^{2}$. If $\sigma^{2}(t)$ is continuous (and we shall assume this) then it has the spectral representation

$$
\sigma^{2}(t)=\int_{-\infty}^{\infty}\left|e^{i \lambda t}-1\right|^{2} \lambda^{-2}\left(1+\lambda^{2}\right) d H(\lambda),
$$

where $H$ is bounded and nondecreasing. For $s<t$ the difference $X(t)-X(s)$ has the stochastic integral representation

$$
X(t)-X(s)=\int_{-\infty}^{\infty} \frac{e^{i \lambda t}-1}{i \lambda} \sqrt{1+\lambda^{2}} \xi(d \lambda),
$$

where $\xi$ is Gaussian with orthogonal increments, and $E|\xi(d \lambda)|^{2}=d H(\lambda)$.

Throughout this work we will assume that $H$ satisfies the following condition: There exist numbers $\alpha$ and $C, 0<\alpha<2, C>0$, such that the derivative $h(\lambda)$ of the absolutely continuous component of $H$ satisfies

$$
|\lambda|^{\alpha-1}\left(1+\lambda^{2}\right) h(\lambda) \geq C, \text { for all sufficiently large }|\lambda| \text {. }
$$

This is equivalent to

$$
|\lambda|^{\alpha+1} h(\lambda) \geq C \text {, for all sufficiently large }|\lambda| \text {. }
$$


We recall the definition of local time. Let $f(t), t \geq 0$, be a real-valued Borel function, and $\mu$ the linear Borel measure. For every pair of Borel sets $A \subseteq(-\infty, \infty)$ and $I \subset[0, \infty)$, define

$$
\nu(A, I)=\mu\left[f^{-1}(A) \cap I\right],
$$

which is the time spent by $f(t), t \in I$, in the set $A$. For fixed $I$, if $\nu(\cdot, I)$ is absolutely continuous, then its derivative $\varphi(x, I)$ is called the local time of $f$ relative to $I$; we then say that the local time exists relative to $I$. We define the function $\varphi(x, t)$ to be $\varphi(x,[0, t])$.

Now let $X(t), t \geq 0$, be a process with Borel sample functions, for example, continuous sample functions. If $X$ is Gaussian then under certain general conditions the local time exists for almost all sample functions [3]. We continue to use the symbol $\varphi(x, t)$ as the local time, but it now depends also on the sample function; therefore, $\varphi(x, t)$ is a stochastic process of two parameters $x, t$, where $-\infty<x<\infty, t \geq 0$.

Here is the outline of the analytic method used in [3] and [5] for the calculation of the moments of $\varphi(x, t)$. For a fixed sample function and fixed $t$, the Fourier transform on $x$ is the function of $u$,

$$
\int_{-\infty}^{\infty} e^{i u x} \varphi(x, t) d x
$$

which is equal to

$$
\int_{0}^{t} e^{i u X(s)} d s .
$$

Express $\varphi(x, t)$ as the inverse Fourier transform of this function:

$$
\varphi(x, t)=\frac{1}{2 \pi} \int_{-\infty}^{\infty} e^{-i u x}\left(\int_{0}^{t} e^{i u X(s)} d s\right) d u .
$$

The $m$ th power of $\varphi$ is

$$
\begin{aligned}
& (2 \pi)^{-m} \int_{-\infty}^{\infty} \cdots \int_{-\infty}^{\infty} \exp \left(-i x \sum_{j=1}^{m} u_{j}\right) \\
& \quad \times \int_{0}^{t} \cdots \int_{0}^{t} \exp \left[i \sum_{j=1}^{m} u_{j} X\left(s_{j}\right)\right] d s_{1} \cdots d s_{m} d u_{1} \cdots d u_{m} .
\end{aligned}
$$

Take the expected value under the sign of integration: the second exponential in the above integral is replaced by the joint characteristic function of $X\left(s_{1}\right), \cdots, X\left(s_{m}\right)$. Interchanging integration and applying the characteristic function inversion formula, we get 


$$
E \varphi^{m}(x, t)=\int_{0}^{t} \cdots \int_{0}^{t} p_{m}\left(x, \cdots, x ; s_{1}, \cdots, s_{m}\right) d s_{1} \cdots d s_{m},
$$

where $p_{m}\left(x_{1}, \cdots, x_{m} ; s_{1}, \cdots, s_{m}\right)$ is the joint density function of $X\left(s_{1}\right), \cdots$, $X\left(s_{m}\right)$ at the point $\left(x_{1}, \cdots, x_{m}\right)$. In the Gaussian case the density is of the form

$$
(2 \pi)^{-m / 2}\left|R\left(s_{1}, \cdots, s_{m}\right)\right|^{-1 / 2} e^{-Q / 2}
$$

where $R$ is the covariance matrix of $X\left(s_{1}\right), \cdots, X\left(s_{m}\right),|R|$ its determinant, and $Q$ a nonnegative quadratic form. Estimates of the moments of $\varphi$ depend on the rate of decrease to 0 of $|R|$ as $s_{j}-s_{j-1} \rightarrow 0$ for some $j$.

In this paper $X(t)$ shall always be a Gaussian process with mean 0 and stationary increments, and have the representations (1.1) and (1.2). In Section $8 X(t)$ will be stationary.

\section{Properties of the finite-dimensional distributions.}

A preliminary result of much use to us is this property of determinants of covariance matrices.

Lemma 2.1 Let $A$ and $B$ be nonnegative definite symmetric matrices of the same order; then $|A| \leq|A+B|$.

Proof. If $|A|=0$, the lemma is trivially true. Now assume $|A|>0$. Let $m$ be the rank of $A$. The well known formula (e.g. [7], p. 120) states that

$$
|A|=(2 \pi)^{m}\left(\int_{R^{m}} \cdots \int \exp \left(-\frac{1}{2} u^{\prime} A u\right) d u\right)^{-2},
$$

where $u$ is an $m$-component column vector. From this relation-with $A+B$ in place of $A-$ and from the linearity and positivity of the quadratic form, we obtain:

$$
\begin{aligned}
|A+B| & =(2 \pi)^{m}\left(\int_{R^{m}} \int e^{-\frac{1}{2} u^{\prime} A u-\frac{1}{2} u^{\prime} B u} d u\right)^{-2} \\
& \geq(2 \pi)^{m}\left(\int_{R^{m}} \cdots \int e^{-\frac{1}{2} u^{\prime} A u} d u\right)^{-2}=|A| .
\end{aligned}
$$

In order to calculate the moments of the local time, we use the following property of the finite-dimensional distributions of the stochastic process (cf. [3], p. 294; [5], p. 1268). We shall first state it, calling it Condition 
$A$, and then prove that it holds under the hypothesis (1.3). The interpretation of this condition is that the successive infinitesimal increments of the process are not too highly correlated.

Condition $A$. For every $T>0$ and positive integer $m$, the determinant of the covariance matrix of the normalized increments

$$
\frac{X\left(t_{j}\right)-X\left(t_{j-1}\right)}{\sigma\left(t_{j}-t_{j-1}\right)}, j=1, \cdots, m, t_{0}=0,
$$

is bounded away from 0 on the $m$-dimensional set

$$
\left\{\left(t_{1}, \cdots, t_{m}\right): 0=t_{0}<t_{1}<\cdots<t_{m} \leq T\right\} .
$$

First we verify Condition $A$ for a special process.

Lemma 2.2. Let $X(t), t \geq 0$, have the particular spectral function

$$
H(\lambda)=\int_{-\infty}^{\lambda} y^{1-\alpha}\left(1+y^{2}\right)^{-1} d y
$$

here $\sigma^{2}$ has the form

$$
\sigma^{2}(t)=K|t|^{\alpha},
$$

where

$$
K=\int_{-\infty}^{\infty}\left|e^{i \lambda}-1\right|^{2}|\lambda|^{-\alpha-1} d \lambda
$$

Then Condition A holds.

Proof. For $0<\alpha<1$, the function (2.3) is concave in $t$, and the conclusion follows from the inequality in [3], p. 1269.

For $1 \leq \alpha<2$, the proof is the same as that of [3], Lemma 5.2. While the latter presumes the stationarity of the process, it is really only the stationarity of the increments that is used in the proof.

In the following two lemmas, Condition $A$ is shown to hold for the more general process.

Lemma 2.3. Under (1.3), for every $T>0$ there exists a constant $b>0$ such that

$$
\sigma^{2}(t) \geq b|t|^{\alpha} \text {, for all } t, 0 \leq t \leq T \text {. }
$$

Proof. For sufficiently large $M, \sigma^{2}(t)$ is, by (1.1) and (1.3), at least equal to 


$$
C \int_{|\lambda|>M}\left|e^{i \lambda t}-1\right|^{2}|\lambda|^{-\alpha-1} d \lambda
$$

This is asymptotic to $C K|t|^{\alpha}$ for $t \rightarrow 0$, and vanishes only for $t=0$.

Lemma 2.4. Condition A holds under (1.3).

Proof. The covariance matrix of (2.1) is representable as the sum of two covariance matrices $A$ and $B$, corresponding to the absolutely continuous and singular parts of $H$, respectively. In proving this lemma, it suffices, by Lemma 2.1 , to consider only the matrix $A$; hence, we shall assume that $H$ is absolutely continuous.

The covariance matrix of (2.1) has another decomposition into matrices of the same type. The stochastic integral (1.2) may be broken up into an integral over $|\lambda| \leq M$ and an integral over $|\lambda|>M$, for arbritary $M>0$. The covariance matrix is the sum of the covariance matrices of these two independent processes. By another application of Lemma 2.1, it suffices to disregard the first matrix and suppose that the function $h(\lambda)$ satisfies

$$
h(\lambda)=0, \text { for }|\lambda| \leq M,
$$

where $M$ is arbitrary but fixed.

Under (1.3) $h(\lambda)$ has another decomposition-into the sum of two nonnegative terms for sufficiently large $|\lambda|$ :

$$
h(\lambda)=\frac{C|\lambda|^{1-\alpha}}{1+\lambda^{2}}+\left(h(\lambda)-\frac{C|\lambda|^{1-\alpha}}{1+\lambda^{2}}\right) .
$$

By another application of Lemma 2.1, we may suppose in the proof that the second term vanishes so that.

$$
h(\lambda)=\frac{C|\lambda|^{1-\alpha}}{1+\lambda^{2}} \text {, for }|\lambda|>M
$$

For simplicity we put $C=1$ :

$$
h(\lambda)=\frac{|\lambda|^{1-\alpha}}{1+\lambda^{2}} \text {, for }|\lambda|>M
$$

As a result of the statements above, the proof of our lemma has now been reduced to that of the case of the function $h$ defined by (2.5) and (2.6). This function differs from that considered in Lemma 2.2 only on the interval $[-M, M]$. We shall complete the proof by showing that this part 
of $h$ has no effect on the nonsingularity of the limiting covariance matrix.

Decompose the vector of normalized increments (2.1) into two independent parts,

$$
\int_{|\lambda| \leq M} \frac{e^{i \lambda t_{j}}-e^{i \lambda t_{j-1}}}{i \lambda \sigma\left(t_{j}-t_{j-1}\right)}\left(1+\lambda^{2}\right)^{1 / 2} \xi(d \lambda)
$$

and

$$
\int_{|\lambda|>M} \frac{e^{i \lambda t_{j}}-e^{i \lambda t_{j-1}}}{i \lambda \sigma\left(t_{j}-t_{j-1}\right)}\left(1+\lambda^{2}\right)^{1 / 2} \xi(d \lambda) .
$$

Let $h$ be the function corresponding to the special process in Lemma 2.2. The determinants of the covariance matrices of the two parts (2.7) and (2.8) are strictly positive on the set (2.2), and the determinant of the sum of the two matrices is bounded away from 0 (Lemma 2.2). This implies that the determinants of (2.7) and (2.8) cannot both tend to 0 along a sequence of points in (2.2) converging to the boundary. Any point on the boundary has equality among at least two successive coordinates $t_{j-1}$ and $t_{j}$; therefore, as a continuous function, positive on (2.2), each determinant is bounded away from 0 if and only if it is so bounded along sequences in (2.2) for which $t_{j}-t_{j-1} \rightarrow 0$ for some $j=1, \cdots, m$. The variance of (2.7) is equal to

$$
K^{-1} \int_{-M}^{M}\left|e^{i \lambda t_{j}}-e^{i \lambda t_{j-1}}\right|^{2}|\lambda|^{-\alpha-1} d \lambda,
$$

and tends to 0 as $t_{j}-t_{j-1} \rightarrow 0$; therefore, the random variable (2.7) converges in probability to 0 as the point in (2.2) approaches the boundary; thus, the portion (2.7) of the process becomes singular at the boundary; therefore, the portion (2.8) cannot become singular. Since the process corresponding to (2.8) has the function $h$ defined by (2.5) and (2.6), the conclusion of Lemma 2.2 holds also for this function; therefore Condition $A$ holds.

We summarize certain results implicit in [3] and [5]:

Lemma 2.5. The determinant of the covariance matrix of

$$
X\left(t_{j}\right)-X(0), j=1, \cdots, m
$$

on the parameter set (2.2) is equal to that of (2.1) times

$$
\prod_{j=1}^{m} \sigma^{2}\left(t_{j}-t_{j-1}\right)
$$


Proof. By elementary row and column operations, the covariance matrix of (2.9) can be transformed into that of $X\left(t_{j}\right)-X\left(t_{j-1}\right), j=1, \cdots, m:$ subtract row $j-1$ from row $j$, and column $j-1$ from column $j$, for $j=2, \cdots$, $m$. (The determinant is unchanged.) Next, divide every element in the $j$ th row by $\sigma\left(t_{j}-t_{j-1}\right)$, and the same for the $j$ th column, $j=1, \cdots, m-1$. The matrix has become the covariance matrix of (2.1). To preserve the original value of the determinant, we multiply the transformed determinant by $(2.10)$.

Lemma 2.6. Under (1.3), for every $T>0$ and positive integer $m$, there exists $B>0$ such that the multiple integral

$$
\begin{gathered}
\int_{t}^{t+h} \cdots \int_{t}^{t+h} \int_{-\infty}^{\infty} \cdots \int_{-\infty}^{\infty} \prod_{j=1}^{m}\left|u_{j}\right|^{\delta} \\
\cdot E\left\{\exp \left[i \sum_{j=1}^{m} u_{j}\left(X\left(t_{j}\right)-X(0)\right)\right]\right\} \prod_{j=1}^{m} d u_{j} \prod_{j=1}^{m} d t_{j}
\end{gathered}
$$

is at most equal to

$$
B|h|^{m\left(1-\frac{\alpha}{2}-\alpha \delta\right)}
$$

for all $\delta$ such that

$$
0 \leq \delta<\frac{1}{\alpha}-\frac{1}{2}
$$

and for all $t$ and $h$ such that $t, t+h \in[0, T]$.

Proof. By Lemma 2.4, Condition $A$ is satisfied; therefore, by the calculations given in [3], p. 294-295 and [5], p. 1268-1269, the integral (2.11) is at most equal to

$$
\left.B^{\prime} \int_{\left\{t=t_{0}<t_{1}<\cdots<t_{m} \leq t+h\right\}} \cdots \int_{j=1}^{m} \sigma\left(t_{j}-t_{j-1}\right)\right]^{-1-\delta} \prod_{j=1}^{m} d t_{j}
$$

where $B^{\prime}$ depends only on $T$ and $m$. (Note that the display in [5], p. 1269, line 9, has two minor errors, and should be corrected to conform to (2.14).) By Lemma 2.3 and elementary integration, we find that $(2.14)$ is at most equal to (2.12), where $B$ is a suitable constant. Note that the exponent in (2.12) is positive because $\delta$ satisfies (2.13). 


\section{One-way Hölder condition for a stochastic process of two parameters.}

In [5, Theorem 5.1] we derived a sufficient condition for the joint continuity of a stochastic process of two parameters. By increasing the strength of the hypothesis, we now get a condition sufficient for a uniform Hölder condition in one variable. As in [5], we shall then apply the result to the local time process.

Theorem 3.1. Let $Y(s, t), 0 \leq s, t \leq 1$, be a stochastic process of two parameters. Suppose there are positive constants $r, b, c, d$ such that:

$$
\begin{gathered}
E|Y(s+h, \tau)-Y(s, \tau)|^{r} \leq b|h|^{1+c}, \\
\qquad \text { for } s, s+h \in[0,1], \tau=0,1 \\
E|Y(s, t+h)-Y(s, t)|^{r} \leq b|h|^{1+d}, \\
\text { for all } t, t+h, s \in[0,1] \\
E|Y(s+k, t+h)-Y(s+k, t)-Y(s, t+h)+Y(s, t)|^{r} \leq b|k|^{1+c}|h|^{1+d} \\
\text { for all } s, s+k, t, t+h \in[0,1] .
\end{gathered}
$$

Then for every $\gamma<d / r$ there exist a version of the process $Y$, and random variables $\eta$ and $\Delta$ which are almost surely positive and finite such that

$$
\begin{aligned}
& |Y(s, t+h)-Y(s, t)| \leq \Delta|h|^{r} \\
& \text { for all } s, t, h \text { satisfying } \\
& s, t, t+h \in[0,1], \text { and }|h|<\eta .
\end{aligned}
$$

Proof. By [5, Theorem 5.1] there is a version of the process with jointly continuous sample functions; indeed, the current hypothesis differs from that of [5] only in that condition (3.2) is stronger. Let $Y$ represent this version of the process.

By (3.3) and a suitable form of the Chebyshev inequality, the series

$$
\begin{gathered}
\sum_{m=1}^{\infty} \sum_{j=1}^{2^{m}} \sum_{n=1}^{\infty} \sum_{k=1}^{2^{n}} P\left\{\mid Y\left(\frac{j}{2^{m}}, \frac{k}{2^{n}}\right)-Y\left(\frac{j-1}{2^{m}}, \frac{k}{2^{n}}\right)\right. \\
\left.-Y\left(\frac{j}{2^{m}}, \frac{k-1}{2^{n}}\right)+Y\left(\frac{j-1}{2^{m}}, \frac{k-1}{2^{n}}\right) \mid>2^{-\frac{m c}{r}-\frac{n d}{r}}\right\}
\end{gathered}
$$

converges; hence, by Borel's Lemma, there exists (random) integers $\nu_{1}$ and $\nu_{2}$ such that 


$$
\begin{gathered}
\max _{1 \leq j \leq 2^{m}, 1 \leq k \leq 2^{n}} \mid Y\left(\frac{j}{2^{m}}, \frac{k}{2^{n}}\right) \\
-Y\left(\frac{j-1}{2^{m}}, \frac{k}{2^{n}}\right)-Y\left(\frac{j}{2^{m}}, \frac{k-1}{2^{n}}\right)+Y\left(\frac{j-1}{2^{m}}, \frac{k-1}{2^{n}}\right) \mid \leq 2^{-\frac{m c}{r}-\frac{n d}{r}}
\end{gathered}
$$

for all $m \geq \nu_{1}$ and $n \geq \nu_{2}$. If $\gamma_{1}<c / r$ and $\gamma_{2}<d / r$, then there exist a constant $D<\infty$ and random variables $\eta^{\prime}$ and $\eta^{\prime \prime}$ which are almost surely positive such that

$$
|Y(s+k, t+h)-Y(s+k, t)-Y(s, t+h)+Y(s, t)| \leq D_{1}|k|^{r_{1}}|h|^{r_{2}}
$$

whenever $|k|<\eta^{\prime}$ and $|h|<\eta^{\prime \prime}$; indeed, this follows from (3.5) by a direct extension of the calculations used to establish the Hölder condition for a process of one parameter (cf. [8], p. 73-74).

The condition (3.2) implies that for each $s \in[0,1]$ and $\gamma_{1}<c / r$, there exist a constant $D_{2}$ and a positive random $\eta$ such that

$$
|Y(s, t+h)-Y(s, t)| \leq D_{2}|h|^{r_{2}}
$$

whenever $|h|<\eta$; this is the content of the theorem just mentioned above. This does not yet complete the proof of (3.4) because the constant $D_{2}$ and the random variable $\eta$ in (3.7) depend on the fixed value of $s$.

We claim that $D_{2}$ is actually independent of $s$. To verify this, note that the condition (3.2) is uniform in $s$, and then use the same proof as that of the one-variable Hölder-condition theorem.

Now we proceed to construct an $\eta$ which is independent of $s$. Let $s$ be an arbitrary number in $[0,1]$, with the dyadic expansion

$$
s=\sum_{p=0}^{\infty} 2^{-p} \alpha_{p}, \text { with } \alpha_{0}=0, \alpha_{p}=0 \text { or } 1 .
$$

It follows from (3.5) and the continuity of $Y$ that $Y(s, t+h)-Y(s, t)$ may be expressed as the absolutely convergent series

$$
\begin{aligned}
& Y(0, t+h)-Y(0, t)+\sum_{n=1}^{\infty}\left[Y\left(\sum_{p=0}^{n} 2^{-p} \alpha_{p}, t+h\right)-Y\left(\sum_{p=0}^{n} 2^{-p} \alpha_{p}, t\right)\right. \\
- & \left.Y\left(\sum_{p=0}^{n-1} 2^{-p} \alpha_{p}, t+h\right)+Y\left(\sum_{p=0}^{n-1} 2^{-p} \alpha_{p}, t\right)\right] .
\end{aligned}
$$

(The absolute convergence follows from the fact that each summand in (3.8) may be expanded in a similar telescoping series with the dyadic representations of $t$ and $t+h$, and that, by (3.5), the double series converges 
absolutely.) Choose $\eta^{\prime}$ and $\eta^{\prime \prime}$ so that (3.6) holds, and then a positive integer $N$ so that $2^{-N}<\eta^{\prime}$; then, the tail of the series (3.8), summed over $n \geq N$, is at most

$$
D_{1}|h|^{r_{2}} \sum_{n=N}^{\infty} 2^{-r_{1} n}
$$

which is not more than

$$
D_{1}\left(\frac{2^{r_{1}}}{2^{r_{1}}-1}\right)|h|^{r_{2}}
$$

whenever $|h|<\eta^{\prime \prime}$.

Let $\eta_{0}$ be such that (3.7) holds for $s=0$ whenever $|h|<\eta_{0}$; and let $\eta_{n}$ be such that the $n$th term of the series (3.8) is dominated by $2 D_{2}|h|^{r_{2}}$ whenever $|h|<\eta_{n}, n=1,2, \cdots$. Such $\eta_{n}$ exist because the $n$th term of the series is at most

$$
\left|Y\left(\sum_{p=0}^{n} 2^{-p} \alpha_{p}, t+h\right)-Y\left(\sum_{p=0}^{n} 2^{-p} \alpha_{p}, t\right)\right|+\left|Y\left(\sum_{p=0}^{n-1} 2^{-p} \alpha_{p}, t+h\right)-Y\left(\sum_{p=0}^{n-1} 2^{-p} \alpha_{p}, t\right)\right| .
$$

As noted above, $D_{2}$ does not depend on $n$. Put

$$
\eta=\min \left(\eta^{\prime \prime}, \eta_{0}, \eta_{1}, \cdots, \eta_{N-1}\right) \text {. }
$$

If $|h|<\eta$, then the sum of the terms of (3.8) up to index $N$ is, by the definition of $\eta_{0}, \eta_{1}, \cdots, \eta_{N-1}$, at most equal to

$$
2 N D_{2}|h|^{r_{2}} \text {. }
$$

Combining (3.9) and (3.10) we find that the series (3.8) is dominated by $\Delta|h|^{r_{2}}$ for $|h|<\eta$, where

$$
\Delta=D_{1}\left(\frac{2^{r_{1}}}{2^{r_{1}}-1}\right)+2 N D_{2}
$$

This completes the proof.

We remark that the conclusion (3.4) differs from the classical Hölder condition for stochastic processes because the bound $\Delta$ here depends on the sample function.

\section{Uniform Hölder condition on the local time}

By Lemma 2.3 the double integral

$$
\int_{0}^{t} \int_{0}^{t} \frac{d s d s^{\prime}}{\sigma\left(s-s^{\prime}\right)}
$$


is finite for every $t>0$; therefore, the local time $\varphi(x, t)$ exists for almost every sample function [3], p. 284. Sufficient conditions on $\sigma^{2}(t)$ for the joint continuity of $\varphi$ are given in [5]. The present condition (1.3) is also sufficient:

Lemma 4.1 Under (1.3) there is a version of the local time which is jointly continuous in $(x, t)$, almost surely.

Proof. Condition $A$ is exactly the hypothesis of [5, Lemma 6.1], which asserts the joint continuity.

We now use (1.3) to get the Hölder condition on $\varphi$ as a function of $t$, uniform in $x$ :

THEOREM 4.1 Under (1.3) there is a jointly continuous version of $\varphi(x, t)$ such that for every pair of intervals $[A, B]$ and $[C, D], C>0$, and every $\gamma<1-\alpha / 2$, there exist random variables $\eta$ and $\Delta$ which are almost surely positive and finite such that

$$
|\varphi(x, t+h)-\varphi(x, t)| \leq \Delta|h|^{r}
$$

for all $x \in[A, B]$, all $t, t+h \in[C, D]$, and all $|h|<\eta$.

Proof. For simplicity we choose $A=C=0$ and $B=D=1$. We identify $\varphi(x, t), 0 \leq x \leq 1,0 \leq t \leq 1$, with the process $Y$ in Theorem 3.1, and show that the conditions of the theorem are satisfied.

For the purpose of the proof we may assume that $X(0)=0$ almost surely. Indeed, consider the process $X(t)-X(0)$ instead of $X(t)$. The local time of $X(t)-X(0)$ is $\varphi(x+X(0), t)$. It is jointly continuous and satisfies the uniform Hölder condition (4.1) if and only if $\varphi(x, t)$, the local time of $X(t)$, does.

For any $\delta, 0 \leq \delta<\frac{1}{\alpha}-\frac{1}{2}$ and positive even integer $m$, the $m$ th moment,

$$
E|\varphi(x+k, t+h)-\varphi(x+k, t)-\varphi(x, t+h)+\varphi(x, t)|^{m}
$$

is, by the method sketched after (1.4) and given in [3], p. 294, at most equal to

$$
\begin{aligned}
& |k|^{m \delta} \pi^{-m} \int_{t}^{t+h} \cdots \int_{t}^{t+h} \int_{-\infty}^{\infty} \cdots \int_{-\infty}^{\infty} \prod_{j=1}^{m}\left|u_{j}\right|^{\delta} \\
& E\left\{\exp \left[i \sum_{j=1}^{m} u_{j} X\left(t_{j}\right)\right)\right\} \prod_{j=1}^{m} d u_{j} \prod_{j=1}^{m} d t_{j} .
\end{aligned}
$$


Since $X(0)=0$, we may apply Lemma 2.6: there is a constant $B=B_{m}$ such that (4.3) is at most

$$
B_{m}|k|^{m \delta}|h|^{\left.m^{\prime} 1-\frac{\alpha}{2}-\alpha \delta\right)} .
$$

By Lemma 4.1 the local time $\varphi(x, t)$ is jointly continuous; therefore, by the definition of $\varphi$, we have

$$
\varphi(x, 0)=0, \text { for all } x .
$$

Now replace $t$ and $t+h$ by 0 and $t$, respectively, in (4.2) and (4.3); then the relation between (4.2) and (4.4) implies

$$
E|\varphi(x+k, t)-\varphi(x, t)|^{m} \leq B_{m}|k|^{m \dot{\delta}} .
$$

From (1.4) and the subsequent discussion we find that $E \mid \varphi(x, t+h)-$ $\left.\varphi(x, t)\right|^{m}$ is a maximum at $x=0$ for fixed $t$ and $h$. Putting $\delta=0$ in (4.3), and using Lemma 2.6, we obtain

$$
E|\varphi(x, t+h)-\varphi(x, t)|^{m} \leq B_{m}|h|^{m\left(1-\frac{\alpha}{2}\right)},
$$

for all $0 \leq x \leq 1$.

In order to apply Theorem 3.1, we now fix the magnitudes of $m$ and $\delta$. For a given $\gamma<1-\alpha / 2$, let $\delta$ be a positive number satisfying

$$
\delta<\frac{1}{\alpha}-\frac{1}{2} \text { and } \gamma<1-\frac{\alpha}{2}-\alpha \delta \text {. }
$$

Choose $m$ (even) so large that each of these inequalities hold:

$$
\begin{aligned}
& m\left(1-\frac{\alpha}{2}-\alpha \delta\right)>1 \\
& m \delta>1 \\
& r<\frac{m\left(1-\frac{\alpha}{2}-\alpha \delta\right)-1}{m} .
\end{aligned}
$$

Put

$$
b=B_{m}, r=m, c=m \delta-1, d=m\left(1-\frac{\alpha}{2}-\alpha \delta\right)-1
$$

these, by (4.7), are positive. With the following correspondence, the conditions of Theorem 3.1 are satisfied: (4.4) represents the bound in (3.3), and the inequalities (4.5) and (4.6) represent (3.1) and (3.2), respectively. The 
conclusion (3.4) follows.

\section{Absence of points where a Hölder condition is satisfied}

For a large class of Gaussian processes the sample functions nowhere satisfy Hölder conditions of given orders [4], and are nowhere differentiable [5], almost surely. Now we shall get sharper results on the lack of Hölder conditions under the hypothesis (1.3).

By extending the method in [5, Lemma 3.1], we obtain this general result about continuous real valued functions and their local times:

Lemмa 5.1 Let $f(t), t \in I$ ( $I$ a real interval), be a continuous function with a jointly continuous local time $\varphi(x, t)$ satisfying a uniform Hölder condition of order $\gamma<1$ :

$$
|\varphi(x, t+h)-\varphi(x, t)| \leq D|h|^{r} \text { for all } x \text {, and } t \in I,
$$

for sufficiently small $h$. Then, for any subinterval $J$ of $I$ :

$$
\max _{J} f(t)-\min _{J} f(t) \geq \frac{\mid \text { length of }\left.J\right|^{1-r}}{\text { constant }} ;
$$

in particular, $f(t)$ nowhere satisfies a Hölder condition of order greater than $1-\gamma$.

Proof. Let $J$ be the interval $[c, d]$. By the definition of the local time, we have:

$$
d-c=\int_{-\infty}^{\infty}[\varphi(x, d)-\varphi(x, c)] d x .
$$

Since $f$ and $\varphi$ are continuous functions, $\varphi(x, d)-\varphi(x, c)$ vanishes for all $x$ outside the range of $f(t), c \leq t \leq d$; therefore, the equation above may be written as

$$
d-c=\int_{\min _{J}}^{\max _{J}}[\varphi(x, d)-\varphi(x, c)] d x .
$$

By the compactness of $I$ we may assume that (5.1) holds for any $h$ such that $t+h \in I$; then, from (5.2), we get

$$
d-c \leq D(d-c)^{r}\left(\max _{J} f-\min _{J} f\right) .
$$

THEOREM 5.1. Under (1.3) the sample function nowhere satisfies a Hölder condition of order larger than $\alpha / 2$, almost surely. 
Proof. There are two cases to consider: when the sample functions are continuous and when they are not.

Suppose first that they are continuous. By Theorem 3.1 the local time satisfies a Hölder condition of order $1-\alpha^{\prime} / 2$ for any $\alpha^{\prime}>\alpha$; therefore, by Lemma 4.1, the sample functions nowhere satisfy a Hölder condition of order greater than $\alpha / 2$.

When not almost surely continuous, Gaussian sample functions are almost surely "badly" discontinuous. Well known theorems describe this property of Gaussian processes (cf. [1], [9]). We want to show that if the sample functions are not almost surely continuous, then neither do they satisfy a Hölder of order greater than $\alpha / 2$ at any point, almost surely. For this purpose, we use the method in [2], p. 193-194. Let $I$ be an arbitrary interval on the positive real axis. According to the reasoning in [2], the spectral representation (1.2) implies that if the sample functions are not almost surely continuous on $I$, then there is a point $\tau$ in $I$ and a positive number $\varepsilon$ such that

$$
\limsup _{t, s \rightarrow \tau} X(t)-X(s) \geq \varepsilon,
$$

almost surely. This must be true almost surely for every $\tau$ in $I$ because (5.3) depends on the increments, which are stationary. It follows that (5.3) must also hold for all $\tau$ in a countable dense subset of $I$, almost surely. Then $X$ cannot satisfy a Hölder condition at any point of $I$; indeed if $|X(t+h)-X(t)| \leq D|h|^{r}$ for all small $h$, then, by the triangle inequality, $\left|X(s)-X\left(s^{\prime}\right)\right| \leq 2 D\left|s-s^{\prime}\right|^{r}$ for all $s$ and $s^{\prime}$ near $t$; in particular, we would have

$$
\sup _{s, s^{\prime}}\left|X(s)-X\left(s^{\prime}\right)\right| \leq \varepsilon / 2
$$

on any interval of length $(\varepsilon / 4 D)^{1 / r}$ and which contains $t$; therefore, (5.3) would not hold for any $\tau$ in such an interval.

6. Lower bound on the dimension of $\{t: X(t)=x\}$.

If the local time of a continuous function $f(t)$ is jointly continuous, then

$$
\{x: f(t)=x \text { for at most countably many } t\}
$$

is nowhere dense in the range of $f[5$, Lemma 3.2]. Using a Hölder condition on the local time we now get a better estimate of the set $\{t: f(t)=$ 
$x$. We first need the following relation between the smoothness of a distribution function and the maximum measure it can assign to small sets.

Lemma 6.1. Let $G(t)$ be a bounded nondecreasing function (distribution function) on an interval $I$, and satisfying a uniform Hölder condition of order $\gamma<1$ at each point. If $B$ is a subset of $I$ of Hausdorf dimension $\beta$, where $\beta<\gamma$, then

$$
\int_{B} d G(t)=0
$$

Proof. By the hypothesis and by the definition of Hausdorff dimension, for every $\gamma^{\prime}>\beta$ and every $n$, there is a covering of $B$ by open sets $I_{n k}, k=$ $1,2, \cdots$ such that

$$
\begin{aligned}
& \text { diameter } I_{n k} \leq 1 / n, \text { for all } k \text {, and } \\
& \lim _{n \rightarrow \infty} \sum_{k=1}^{\infty} \mid \text { diameter }\left.I_{n k}\right|^{r \prime}<\infty .
\end{aligned}
$$

If $\gamma^{\prime}<\gamma$, then

$$
\lim _{n \rightarrow \infty} \sum_{k=1}^{\infty} \mid \text { diameter }\left.I_{n k}\right|^{r}=0 \text {. }
$$

For any $\beta<\gamma$, choose $\gamma^{\prime}$ so that $\beta<\gamma^{\prime}<\gamma$; then, by the limit relation above and by the uniform Hölder condition on $G$, we have

$$
\begin{aligned}
\int_{B} d G(t) & \leq \limsup _{n \rightarrow \infty} \sum_{k=1}^{\infty} \int_{I_{n k}} d G(t) \\
& \leq \text { constant } \cdot \lim _{n \rightarrow \infty} \sum_{k=1}^{\infty} \mid \text { diameter }\left.I_{n k}\right|^{r}=0 .
\end{aligned}
$$

The next lemma concerns the dimension of $\{t: f(t)=x\}$ when $f$ is continuous, with a jointly continuous local time satisfying a Hölder condition.

LemmA 6.2. If $f(t), 0 \leq t \leq T$, is continuous, and its local time is jointly continuous and satisfies a Hölder condition

$$
|\varphi(x, t+h)-\varphi(x, t)| \leq D|h|^{r}
$$

uniformly in $(x, t)$ for all sufficiently small $h$, then the set

$$
\{x: \operatorname{dim}[t: 0 \leq t \leq T, f(t)=x]<\beta\}
$$

is included in the set of zeros of $\varphi(x, T)$ whenever $\beta \leq \gamma$.

Proof. When the local time $\varphi(x, t)$ is jointly continuous, it is a distribu- 
tion function in $t$ for every $x$; furthermore, when $f$ is continuous, then for each $x$ the support of the distribution $\varphi(x, t), 0 \leq t \leq T$, is contained in $\{t$ : $0 \leq t \leq T, f(t)=x\}$ [5, Lemma 1.5]. If this set has dimension smaller than $\beta$, then $\varphi(x, T)=0$ because $\varphi$ satisfies a Hölder condition of order $\gamma$, and, by Lemma 6.1, cannot assign positive measure to a subset of dimension smaller than $r$.

This is applied to the sample functions of the stochastic process:

Theorem 6.1. If $X(t)$ has continuous sample functions, and if (1.3) holds, then the set

$$
\left\{x: \operatorname{dim}[t: 0 \leq t \leq T, X(t)=x]<1-\frac{\alpha}{2}\right\}
$$

is nowhere dense, almost surely.

Proof. The local time is jointly continuous (Lemma 4.1) and satisfies a uniform Hölder condition of any order $\gamma<1-\alpha / 2$ (Theorem 4.1). For any $\beta<1-\alpha / 2$, choose $\gamma$ so that $\beta<\gamma<1-\alpha / 2$. By Lemma 6.2, the set

$$
\{x: \operatorname{dim}[t: 0 \leq t \leq T, X(t)=x]<\beta\}
$$

is included in the set of zeros of the local time. By [3, Lemma 2.6] this set is nowhere dense in the range of $X$. Since (6.2) is a subset of the range it is nowhere dense.

\section{Upper bound on the dimension of $\{t: X(t)=x\}$.}

The condition (1.3) guarantees a significant contribution to the process from the high-frequency components of the spectrum. These components are very irregular: their "scattering" effect gives most points in the range an inverse image of positive dimension. This is why (1.3) implies a lower bound on the dimension.

In order to derive an upper bound on the dimension, we put an upper bound on the contribution of the high-frequency components. A condition of the following kind is sufficinnt: $H(\lambda)$ is absolutely continuous for all large $|\lambda|$, and its derivative $h(\lambda)$ satisfies a condition of the type (1.3) with the inequality sign reversed:

$$
|\lambda|^{\beta-1}\left(1+\lambda^{2}\right) h(\lambda) \leq B \text {, for all sufficiently large }|\lambda| \text {, }
$$

for some $\beta, 0<\beta<2$.

Lemma 7.1. Under (1.7), for every $T>0$, there exists $B>0$ such that 


$$
\sigma^{2}(t) \leq B|t|^{\beta}, \text { for } 0 \leq t \leq T .
$$

Proof. The integral (1.1) is the sum of a finite part-over $|\lambda| \leq M$-and an infinite part-over $|\lambda|>M$. The finite part is of the order $t^{2}$ for $t \rightarrow 0$ :

$$
\int_{-M}^{M}\left|e^{i \lambda t}-1\right|^{2} \lambda^{-2}\left(1+\lambda^{2}\right) d H(\lambda) \leq t^{2} \int_{-M}^{M}\left(1+\lambda^{2}\right) d H(\lambda) .
$$

Using the proof of Lemma 2.3 with the inequality reversed, we find that the infinite part is of the order $|t|^{\beta}$ for $t \rightarrow 0$.

According to [5, Theorem 2.1] the condition $\sigma^{2}(t) \sim C|t|^{\beta}$ implies that $\operatorname{dim}\{t: 0 \leq t \leq T, X(t)=x\} \leq 1-\beta / 2$, almost surely for each $x$. An examination of the proof shows that the weaker condition (7.2) is sufficient; actually, $\sigma^{2}(t) \sim C|t|^{\beta}$ was used to get an exact estimate of the dimension. Now we show that the weaker hypothesis (7.2) furnishes a lower bound for the dimension which holds for all $x$, almost surely.

We begin with a result on real valued functions:

Lemma 7.2. Let $f(t), 0 \leq t \leq 1$, satisfy a uniform Hölder condition of every order smaller than $p, 0<p<1$, and have a jointly continuous local time. If $x$ is not equal to any of the values

$$
f\left(k 2^{-n}\right), \text { for } k=0,1, \cdots, 2^{n}, n=1,2, \cdots,
$$

then

$$
\operatorname{dim}\{t: 0 \leq t \leq 1, f(t)=x\} \leq 1-p .
$$

Proof. Let $I_{n k}$ be the open interval $\left((k-1) 2^{-n}, k 2^{-n}\right)$. If $x$ is not one of the values (7.3), then, for every $n \geq 1$, the intervals $I_{n k}$ containing at least one point of the set $\{t: f(t)=x\}$ form an open covering of this set. For any $r, 0<\gamma<1$, the sum of the $\gamma$ th powers of the lengths of the intervals in the covering is

$$
\begin{aligned}
& 2^{-n \gamma} \cdot \text { number of intervals } I_{n k}, 1 \leq k \leq 2^{n} \text {, such that } \\
& f(t)=x \text { for some } t \text { in } I_{n k} .
\end{aligned}
$$

By hypothèsis, for every $\delta<p$, there exists $D>0$ such that

$$
|f(t)-f(s)| \leq D|t-s|^{\delta}, \text { for } s, t \in I_{n k}, k=1, \cdots, 2^{n},
$$

for all sufficiently large $n$; thus, for all large $n,(7.4)$ is not more than

$$
\begin{aligned}
& 2^{-n r} \text { number of intervals } I_{n k} \text { such that }|f(t)-x| \leq D 2^{-n \delta} \\
& \text { for all } t \text { in } I_{n k}, 1 \leq k \leq 2^{n} \text {. }
\end{aligned}
$$


We claim that (7.5) tends to 0 if $r>1-\delta$. Assume the contrary. For $\varepsilon>0$, let (7.5) exceed $\varepsilon$ for infinitely many $n$. Then the time spent by $f(s), 0 \leq s \leq 1$, in the interval $x \pm D 2^{-n \delta}$ exceeds $\varepsilon 2^{-(1-r)}$ for infinitely many $n$; thus, by the definition of the local time, the inequality

$$
2^{n(1-r)} \int_{x-D 2^{-n \delta}}^{x+D 2^{-n \delta}} \varphi(y, 1) d y \geq \varepsilon
$$

holds for infinitely many $n$. But this contradicts the continuity of $\varphi$ :

$$
2^{n(1-r)} \int_{x-D 2^{-n \delta}}^{x+D 2^{-n \delta}} \varphi(y, 1) d y \leq 2 D \max _{0 \leq y \leq 1} \varphi(y, 1) 2^{-n(r+\delta-1)} \rightarrow 0 .
$$

Since $\delta$ is an arbitrary number smaller than $p$, the conclusion of the lemma holds.

In the proof of the dimension theorem for the stochastic process, we do not need the full strength of the condition (7.1) but only its implication (7.2).

THEOREM 7.1. If (1.3) and (7.2) hold, then

$$
\operatorname{dim}\{t: 0 \leq t \leq T, X(t)=x\} \leq 1-\beta / 2
$$

for all $x$, almost surely. (The particular value of $\alpha$ in (1.3) is unimportant as long as $0<\alpha<2$.)

Proof. For simplicity, take $T=1$. Under (7.2) $X(t)$ satisfies a uniform Hölder condition of order $\delta$, for any $\delta<\beta / 2$, almost surely [8], p. 172 . The hypothesis of Lemma 7.2 is fulfilled; thus, (7.6) holds for all $x$ not of the form $X\left(k 2^{-n}\right), k=0,1, \cdots, 2^{n} ; n=1,2, \cdots$, almost surely.

Next, we have to prove that (7.6) is satisfied whenever $x=X\left(k 2^{-n}\right)$ for some $k$ and $n$. It suffices to show that

$$
\operatorname{dim}\{t: X(t)=X(\tau), 0 \leq t \leq 1\} \leq 1-\beta / 2
$$

almost surely for each $\tau$. The proof of this is similar to that of [5, Theorem 2.1, first part]: Substitute $X(\tau)$ for $x$, and use the estimates

$$
\begin{aligned}
& 2^{-n r} \sum_{k=1}^{2^{n}} P\left[\left|X\left(k 2^{-n}\right)-X(\tau)\right| \leq D 2^{-n \delta}\right] \\
\leq & 2^{-n(\gamma+\delta-1)}(2 / \pi)^{1 / 2} 2^{-n} \sum_{k=1}^{2^{n}}\left(\sigma\left(\tau-k 2^{-n}\right)\right)^{-1},
\end{aligned}
$$

and (from (2.4)) 


$$
\begin{gathered}
2^{-n} \sum_{k=1}^{2^{n}}\left(\sigma\left(\tau-k 2^{-n}\right)\right)^{-1} \leq b^{-\frac{1}{2}} 2^{-n} \sum_{k=1}^{2^{n}}\left|\tau-k 2^{-n}\right|^{-\frac{\alpha}{2}} \\
\sim b^{-\frac{1}{2}} \int_{0}^{1}|\tau-s|^{-\alpha / 2} d s .
\end{gathered}
$$

\section{Uniform Hausdorff dimension for all real values for sta- tionary Gaussian processes}

Now let $X$ be stationary, not just have stationary increments. For simplicity the mean is assumed to be 0 and the variance 1 . Let the covariance function $r(t)$ be continuous, with the spectral representation

$$
r(t)=\int_{-\infty}^{\infty} e^{i \lambda t} d F(\lambda)
$$

where $F$ is the spectral distribution function; then

$$
\sigma^{2}(t)=2(1-r(t)) .
$$

$F$ is related to $H$ through the equation

$$
d F(\lambda)=\lambda^{-2}\left(1+\lambda^{2}\right) d H(\lambda) .
$$

The condition (1.3) on $H$ is equivalent to the condition on $F$ :

$$
|\lambda|^{\alpha+1} F^{\prime}(\lambda) \geq C \text {, for all sufficiently large }|\lambda|,
$$

where $F^{\prime}$ is the derivative of the absolutely continuous part of $F$. Recall that $X$ is ergodic if and only if $F$ is continuous [8], p. 157.

We now consider the $x$-values of $X(t)$ for $0 \leq t<\infty$, not just for $t$ on a finite interval. The lower uniform estimate of the dimension is based on

Lemma 8.1. If $X$ is ergodic, and (8.3) holds, then

$$
\limsup _{t \rightarrow \infty} t^{-1} \varphi(x, t)>0
$$

for all $x$, almost surely.

Proof. Let $\varphi(x)$ be the standard Gaussian density. The idea of the proof is that the family of processes $\left\{t^{-1} \varphi(x, t):-\infty<x<\infty\right\}$ "converges", for $t \rightarrow \infty$, to the nonrandom process $\varphi(x)$. Since $\varphi(x)>0$ for all $x$, we conclude that (8.4) holds for all $x$. Our program is to show that the convergence

$$
t^{-1} \varphi(x, t) \rightarrow \varphi(x) \text {, for } t \rightarrow \infty,
$$

is sufficiently uniform in $x$. For this purpose we shall show that (8.5) holds 
in the sense of weak convergence of measures over the space of continuous functions of $x$.

For fixed $x$, the stochastic process $\varphi(x, t), t \geq 0$, has stationary increments because $X$ is stationary; therefore, by the ergodic theorem,

$$
\lim _{t \rightarrow \infty} t^{-1} \varphi(x, t)
$$

exists almost surely. The limit is constant because $X$ is ergodic. By the formula (1.5) with $m=1$, we have

$$
E\left[t^{-1} \varphi(x, t)\right]=\varphi(x), \text { for } t>0 ;
$$

therefore, (8.5) holds almost surely, for each $x$. It follows immediately that the convergence (8.5) also holds in the sense of finite-dimensional distributions: the process $\left\{t^{-1} \varphi(x, t),-\infty<x<\infty\right\}$ converges in distribution to the (nonrandom) process $\{\varphi(x),-\infty<x<\infty\}$.

Next we show: For every closed bounded interval $J$ on the real line, the convergence (8.5) for $x \in J$ is weak convergence of the induced probability measures on $\boldsymbol{C}(J)$. There are two conditions to verify. (See [6], p. 95). Since (8.6) has already been demonstrated, it suffices to prove: there exists constants $D>0$ and $c>0$, and integer $m>0$ such that

$$
E\left|t^{-1} \varphi(x, t)-t^{-1} \varphi\left(x^{\prime}, t\right)\right|^{m} \leq D\left|x-x^{\prime}\right|^{1 \div c}
$$

for all $x, x^{\prime}$ and $t$. From (1.4) and the calculations used to derive (4.3), we find that the left hand side of (8.7) is at most

$$
\begin{aligned}
& \pi^{-m}\left|x-x^{\prime}\right|^{m \delta} t^{-m} \int_{0}^{t} \cdots \int_{0}^{t} \int_{-\infty}^{\infty} \cdots \int_{-\infty}^{\infty} \prod_{j=1}^{m}\left|u_{j}\right|^{\delta} \\
& E\left\{\exp \left[i \sum_{j=1}^{m} u_{j} X\left(t_{j}\right)\right]\right\} \prod_{j=1}^{m} d u_{j} \prod_{j=1}^{m} d t_{j},
\end{aligned}
$$

where $\delta<\frac{1}{\alpha}-\frac{1}{2}$. Change variables of integration from $t_{j}$ to $t t_{j}, j=1$, $\cdots, m$, and estimate the integral as in [3], p. 294: (8.8) is bounded above by a constant (which is independent of $x, x^{\prime}$, and $t$ ) multiple of

$$
\left|x-x^{\prime}\right|^{m \delta} \int_{\left\{0=t_{0}<\cdots<t_{m} \leq 1\right\}} \cdots \int_{j=1}\left[\prod_{j}^{m} \sigma\left(t\left(t_{j}-t_{j-1}\right)\right)\right]^{-1-\delta} \prod_{j=1}^{m} d t j .
$$

(This is a slight variant of the computation leading to (2.14).) Under (8.3), $\sigma^{2}(t)$ vanishes only for $t=0$, and is bounded away from 0 on every closed subinterval of the positive $t$-axis; hence, by Lemma 2.3 , the coefficient of 
$\left|x-x^{\prime}\right|^{m \delta}$ in (8.9) is bounded for $t \rightarrow \infty$. For any $\delta<\frac{1}{\alpha}-\frac{1}{2}$, choose $m$ so that $m \delta>1$; then (8.7) holds with $c=m \delta-1$.

From the weak convergence relation (8.5) and the linearity of such convergence it follows that the process

$$
\left\{t^{-1} \varphi(x, t)-\varphi(x), x \in J\right\}
$$

converges weakly, for $t \rightarrow \infty$, to the process identically equal to 0 on $J$; therefore, the distribution of

$$
\max _{x \in J}\left|t^{-1} \varphi(x, t)-\varphi(x)\right|
$$

converges to the distribution of the maximum of the process identically equal to 0 ; hence,

$$
\max _{x \in J}\left|t^{-1} \varphi(x, t)-\varphi(x)\right| \rightarrow 0, \text { in probability, }
$$

for $t \rightarrow \infty$, and so there exists a sequence $t_{n} \rightarrow \infty$ such that $\max \mid t_{n}^{-1} \varphi\left(x, t_{n}\right)$ $-\varphi(x) \mid \rightarrow 0$, almost surely.

Since $\varphi(x)>0$ for all $x$, it follows that (8.4) holds for every $x \in J$, almost surely. It then holds for all $x$, almost surely, because $J$ is arbitrary.

Now we combine the result of Lemma 8.1 with the upper uniform dimension estimate of Theorem 7.1:

Theorem 8.1. Let $X(t)$ be stationary and ergodic, and satisfying (7.2) and (8.3) with $\alpha=\beta$. (The former is assumed to hold for some $T>0$.) Then

$$
\operatorname{dim}\{t: t \geq 0, X(t)=x\}=1-\alpha / 2
$$

for all $x$, almost surely.

Proof. Suppose, for some $x$ and some sample function $X$,

$$
\operatorname{dim}\{t: t \geq 0, X(t)=x\}<1-\alpha / 2 ;
$$

then, for every $T>0$,

$$
\operatorname{dim}\{t: 0 \leq t \leq T, X(t)=x\}<1-\alpha / 2 .
$$

Under (7.2), $X$ is continuous almost surely; and, under (8.3), the local time is jointly continuous and satisfies a uniform Hölder condition of every order smaller than $1-\alpha / 2$, on the interval $[0, T]$. By Lemma 6.2, this particular value of $x$ satisfies $\varphi(x, T)=0$. Since $T$ is arbitrary, it follows that $t^{-1} \varphi(x$, $t)=0$ for all $t>0$. By Lemma 8.1, this occurs for some $x$ with at most 
probability 0 ; therefore

$$
\operatorname{dim}\{t: t \geq 0, X(t)=x\} \geq 1-\alpha / 2
$$

for all $x$, almost surely.

If (7.2) holds for some $T>0$, and (8.3) holds with $\alpha=\beta$, then, by Theorem, 7.1,

$$
\operatorname{dim}\{t: 0 \leq t \leq T, X(t)=x\} \leq 1-\alpha / 2
$$

for all $x$, almost surely. By stationarity this holds when the $t$-set $[0, T]$ is arbitrarily translated along the $t$-axis; therefore, this continues to hold for $T=\infty$ :

$$
\operatorname{dim}\{t: t \geq 0, X(t)=x\} \leq 1-\alpha / 2
$$

for all $x$, almost surely. Combining this with (8.12), we obtain (8.11).

We remark that the conditions of the theorem are satisfied if $F$ is absolutely continuous and $F^{\prime}$ satisfies $B_{1} \leq|\lambda|^{\alpha+1} F^{\prime}(\lambda) \leq B_{2}<\infty$ for all sufficiently large $|\lambda|$, for some $B_{1}$ and $B_{2}$.

\section{The special case of Brownian Motion}

The methods of Sections 7 and 8 give an exact uniform dimension of $x$-values in the particular case of the Brownian motion process. Here (1.3) and (7.2) hold with $\alpha=\beta=\frac{1}{2}$. According to the proof of Theorem 6.1, $\varphi(x, T)$ vanishes whenever $x$ belongs to the set (6.2). It is shown in [12] that the local time of Brownian motion is positive on the interior of the range of $X$; thus, no point in (6.2) belongs to the interior of range. Combining this with Theorem 7.1, we obtain:

$$
\operatorname{dim}\{t: 0 \leq t \leq T, X(t)=x\}=\frac{1}{2}
$$

for every $x$ in the interior of the range of $X(t), 0 \leq t \leq T$, almost surely. The only previous results on exact dimension for $x$-values of Brownian motion appear to hold only for a fixed value of $x$ (See [10] and [13].)

\section{REFERENCES}

[1] Ju.K. Beljaev, Continuity and Hölder's conditions for sample functions of stationary Gaussian processes, Proc. Fourth Berkeley Sympos. Math. Stat. and Prob., Vol. 2: Contributions to probability theory, Univ. of California Press, Berkeley, Calif., 1961, pp. 23-33.

[2] S.M. Berman, Some continuity properties of Brownian motion with the time parameter in Hilbert 
space, Trans. Amer. Math. Soc. 131 (1968), 182-198.

[ 3 ] S.M. Berman, Local times and sample function properties of stationary Gaussian processes, Trans. Amer. Math. Soc. 137 (1969), 277-299.

[4] S.M. Berman, Harmonic analysis of local times and sample functions of Gaussian processes, Trans. Amer. Math. Soc. 143 (1969), 269-281.

[5] S.M. Berman, Gaussian processes with stationary increments: local times and sample function properties, Ann. Math. Statist. 41 (1970), 1260-1272.

[ 6 ] P. Billingsley, Convergence of Probability Measures, John Wiley, New York, 1968.

[ 7 ] H. Cramér, Mathematical Methods of Statistics, Princeton Univ. Press, Princeton, 1946.

[ 8 ] H. Cramér and M.R. Leadbetter, Stationary and Related Stochastic Processes, John Wiley, New York, 1967.

[9] R.M. Dudley, The sizes of compact subsets of Hilbert space and continuity of Gaussian processes, J. Functional Analysis 1 (1967), 290-330.

[10] K. Ito and H.P. McKean, Jr., Diffusion Processes and their Sample paths, Springer-Verlag, Berlin, 1965.

[11] S. Orey, Gaussian sample functions and the Hausdorff dimension of level crossings, Z. Wahrscheinlichkeitstheorie verw. Geb. 17 (1971), 39-47.

[12] Ray, D.B., Sojurn times of diffusion processes, Illinois J. Math. 7 (1963),615-630.

[13] Taylor, S.J., The $\alpha$-dimensional measure of the graph and the set of zeros of a Brownian path, Proc. Cambridge Philos. Soc. 51 Part II (1955), 265-274.

New York University 